\title{
Polyphosphoric Acid Trimethylsilyl Ester (PPSE) Promoted Intramolecular Acylation of an Olefin by a Carboxylic Acid: Convenient Construction of C-18 Functionalized $\square^{14}$-Hecogenin Acetate
}

\author{
Wei Li and Philip L. Fuchs* \\ Contribution from the Department of Chemistry, Purdue University, \\ West Lafayette, Indiana 47907
}




\section{Compound characterization checklist.}

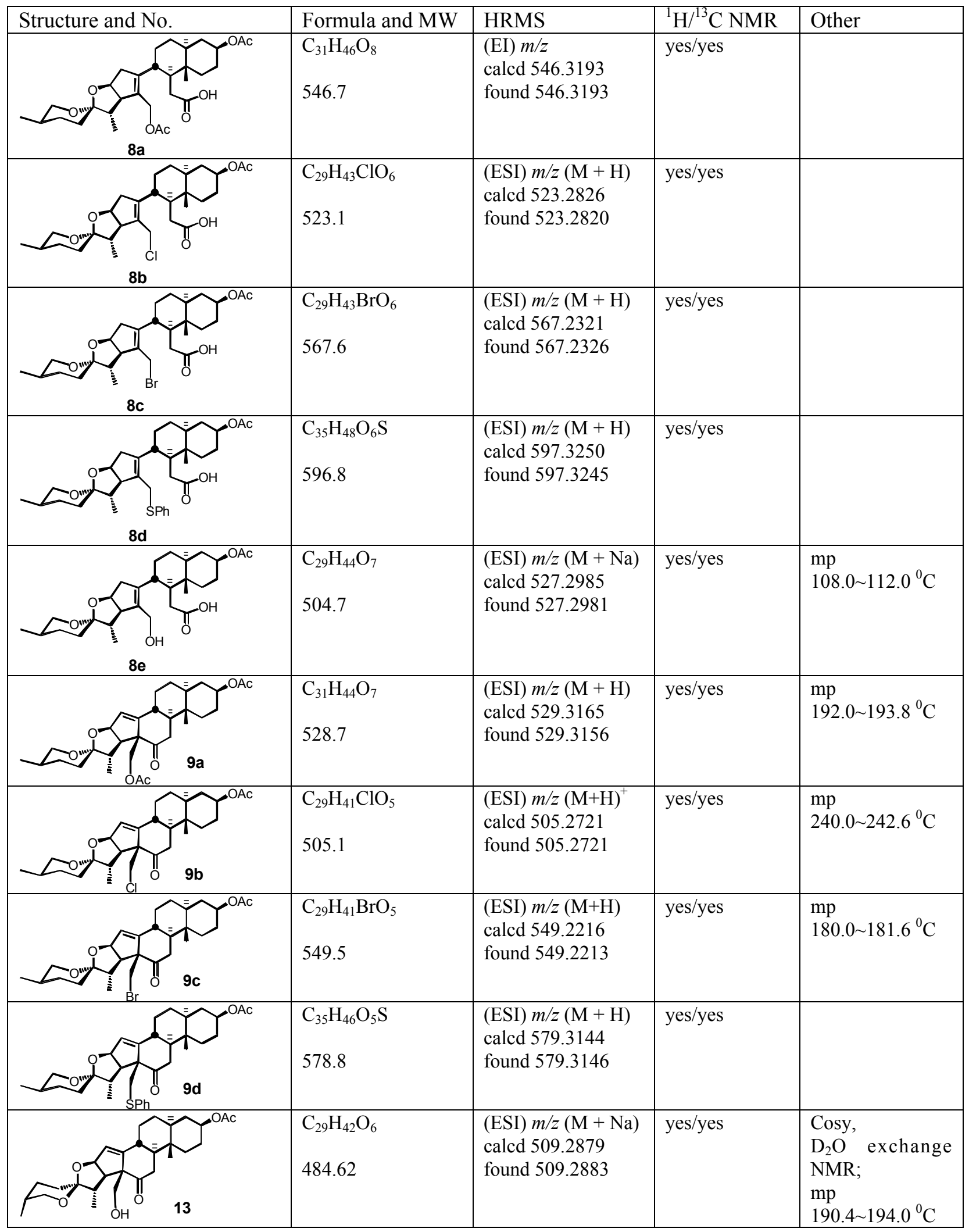


Materials and general methods.

Unless otherwise stated, reactions were performed in glassware recently removed from the oven and cooled under a nitrogen atmosphere. All reactions employed freshly distilled solvents. Methylene chloride, acetonitrile, toluene and benzene were distilled from calcium hydride. Tetrahydrofuran and diethyl ether were distilled from sodium with benzophenone. All other commercially obtained reagents were used as received. Ingredients are listed in the order of their addition. All reactions were magnetically stirred (unless otherwise stated) and monitored by thin-layer chromatography (TLC) using cut EM Si gel 60 F-254 $(0.25 \mathrm{~mm})$ plates, visualized with a UV lamp (254 nm) and p-anisaldehyde (for TLC colors, stain solution: EtOH, $1350 \mathrm{~mL}$, $\mathrm{H}_{2} \mathrm{SO}_{4} 50 \mathrm{~mL}, \mathrm{AcOH}, 15 \mathrm{~mL}$, p-anisaldehyde $37 \mathrm{~mL}$; heat activated).

Standard work up: the reaction was diluted with organic solvent and washed with saturated $\mathrm{NaHCO}_{3}$ or $5 \% \mathrm{HCl}$ solution, then washed with brine, dried with anhydrous $\mathrm{Na}_{2} \mathrm{SO}_{4}$ and decanted. Solvent was removed under reduced pressure using a rotovap and then high vacuum ("concentrated" or "stripped"). Silica gel column chromatography ("sgc") with 230-400 mesh Si gel was based on the method of Still. ${ }^{1}$

All melting points were obtained on a MEL-TEMP and are uncorrected. ${ }^{1} \mathrm{H}$ and ${ }^{13} \mathrm{C}$ NMR spectra were determined on Varian INOVA-300 and Bruker DRX-500 spectrometers. High resolution mass spectra were performed by the Purdue Campus-wide Mass Spectrometry Facility. Single crystal X-ray analysis was performed by Dr. Fanwick at Purdue University.
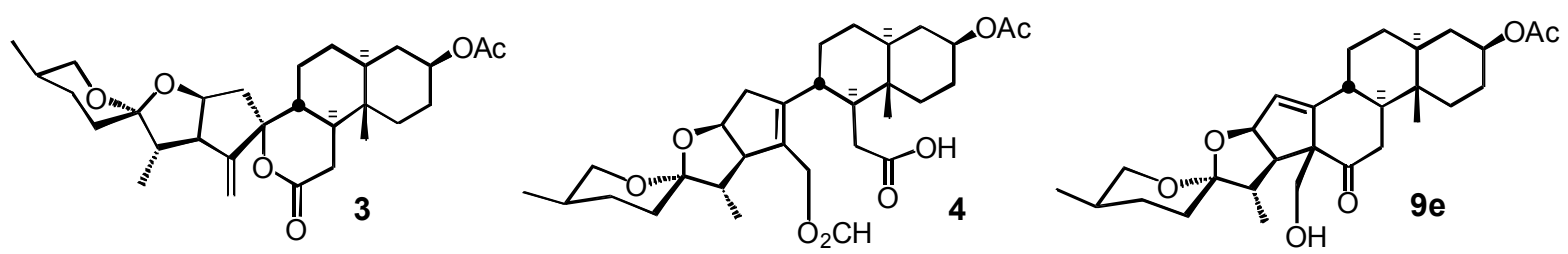

\section{Preparation of 8a:}

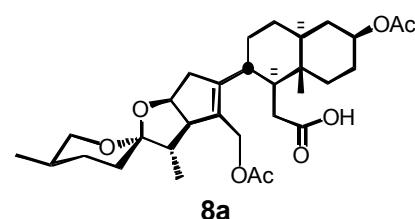

To a mixture of acetic acid $(0.5 \mathrm{~mL})$ and sulfuric acid (1 $\square \mathrm{L})$ was added compound 3 (100 mg, $0.206 \mathrm{mmol})$. The solution was stirred at room temperature for 10 hours. The reaction solution was then poured on ice and extracted with diethyl ether. The ether layers were combined and washed successively with water, cold aqueous bicarbonate, and brine. After drying over anhydrous sodium sulfate, the solvent was removed and the remainder was chromatographed (hexanes/ethyl acetate) to give a white solid (107 mg, $\mathrm{y}$ $=96 \%$, containing $97 \%$ of $\mathbf{8 a}$ and $3 \%$ of the starting material 3 by ${ }^{1} \mathrm{H} \mathrm{NMR}$ ). This material is directly used for the next step without further purification.

(8a) ${ }^{1} \mathrm{H}$ NMR $\left(300 \mathrm{~Hz}, \mathrm{CDCl}_{3}\right)$ : $\square$ 4.68-4.64 (2H, m, $\mathrm{H}_{18 \mathrm{a}}$ and $\left.\mathrm{H}_{16}\right), 4.52-4.46(2 \mathrm{H}, \mathrm{m}$, $\mathrm{H}_{18 \mathrm{~b}}$ and $\left.\mathrm{H}_{3}\right), 3.42\left(1 \mathrm{H}, \mathrm{dd}, \mathrm{J}=9.9 \mathrm{~Hz}, 3.8 \mathrm{~Hz}, \mathrm{H}_{26}\right), 3.34\left(1 \mathrm{H}\right.$, apt, J = $\left.10.7 \mathrm{~Hz}, \mathrm{H}_{26}\right), 2.91(1 \mathrm{H}$, apt, $\left.\mathrm{J}=7.3 \mathrm{~Hz}, \mathrm{H}_{17}\right), 2.58\left(1 \mathrm{H}, \mathrm{dd}, \mathrm{J}=17.9,7.5 \mathrm{~Hz}, \mathrm{H}_{11 \square}\right), 2.54-2.45(1 \mathrm{H}, \mathrm{m}), 2.35(1 \mathrm{H}, \mathrm{d}, \mathrm{J}=$ $\left.17.7 \mathrm{~Hz}, \mathrm{H}_{11 \square}\right), 2.21(1 \mathrm{H}, \mathrm{dd}, \mathrm{J}=17.0,6.3 \mathrm{~Hz}), 2.01\left(3 \mathrm{H}, \mathrm{s}, \mathrm{H}_{18 \mathrm{OAc}} 1.97\right.$ (3H, s, H3OAc), 1.01 (3H, $\left.\mathrm{d}, \mathrm{J}=6.9 \mathrm{~Hz}, \mathrm{H}_{21}\right), 0.80\left(3 \mathrm{H}, \mathrm{s}, \mathrm{H}_{19}\right), 0.75\left(3 \mathrm{H}, \mathrm{d}, \mathrm{J}=6.3 \mathrm{~Hz}, \mathrm{H}_{27}\right) ;{ }^{13} \mathrm{C} \mathrm{NMR}\left(500 \mathrm{~Hz}, \mathrm{CDCl}_{3}\right): \square$ 179.4, 171.1, 170.6, 142.8, 133.4, 106.1, 77.5, 73.1, 66.8, 59.2, 58.4, 48.6, 46.3, 43.7, 37.7, 37.6,

\footnotetext{
${ }^{1}$ Still, W. C., Kahn, M., Mitra, A. J. Org. Chem. 1978, 43, 2923.
} 
36.6, 36.4, 33.8, 33.3, 31.1, 31.0, 30.2, 28.6, 27.8, 27.0, 21.3, 20.8, 17.0, 13.6, 11.8; HRMS (EI) $\mathrm{m} / \mathrm{z}$ : calcd 546.3193, found 546.3193 .

\section{Preparation of $\mathbf{8 b}$ :}

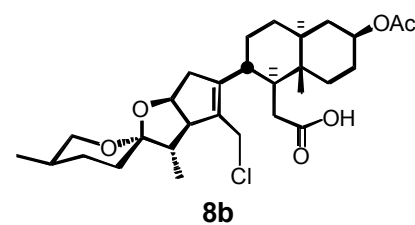

To a solution of compound 3 (100 $\mathrm{mg}, 0.206 \mathrm{mmol})$ in dichloromethane $(1.0 \mathrm{~mL})$ was added hydrogen chloride etherate $(0.6$ $\mathrm{mL}, 0.6 \mathrm{mmol})$. After stirring at room temperature for 2 hours, the solution was quenched with saturated aqueous sodium bicarbonate and diluted with ethyl acetate. The organic layer was washed with brine and dried over anhydrous sodium sulfate. The solvent was removed and the residue was chromatographed (hexanes/ethyl acetate 2:1, 1:1) to give compound $\mathbf{8 b}$ (106 $\mathrm{mg}, \mathrm{y}=98 \%)$ as a white solid.

${ }^{1} \mathrm{H}$ NMR $\left(300 \mathrm{~Hz}, \mathrm{CDCl}_{3}\right): \square 4.68-4.66\left(1 \mathrm{H}, \mathrm{m}, \mathrm{H}_{3}\right), 4.52\left(1 \mathrm{H}, \mathrm{apt}, \mathrm{J}=6.7 \mathrm{~Hz}, \mathrm{H}_{16}\right), 4.16$ $\left(1 \mathrm{H}, \mathrm{d}, \mathrm{J}=11.5 \mathrm{~Hz}, \mathrm{H}_{18 \mathrm{a}}\right), 4.01\left(1 \mathrm{H}, \mathrm{d}, \mathrm{J}=11.5 \mathrm{~Hz}, \mathrm{H}_{18 \mathrm{~b}}\right), 3.46(1 \mathrm{H}, \mathrm{dd}, \mathrm{J}=10.1 \mathrm{~Hz}, 3.2 \mathrm{~Hz}$, $\left.\mathrm{H}_{26 \square}\right), 3.37\left(1 \mathrm{H}\right.$, apt, $\left.\mathrm{J}=10.7 \mathrm{~Hz}, \mathrm{H}_{26}\right), 3.06\left(1 \mathrm{H}\right.$, apt, $\left.\mathrm{J}=7.3 \mathrm{~Hz}, \mathrm{H}_{17}\right), 2.62(1 \mathrm{H}, \mathrm{dd}, \mathrm{J}=18.2$, $\left.7.5 \mathrm{~Hz}, \mathrm{H}_{11 \square}\right), 2.46-2.41(1 \mathrm{H}, \mathrm{m}), 2.38\left(1 \mathrm{H}, \mathrm{d}, \mathrm{J}=18.2 \mathrm{~Hz}, \mathrm{H}_{11}\right), 2.25(1 \mathrm{H}, \mathrm{dd}, \mathrm{J}=17.1,6.9 \mathrm{~Hz})$, $1.99\left(3 \mathrm{H}, \mathrm{s}, \mathrm{H}_{3 \mathrm{OAc}}\right), 1.01\left(3 \mathrm{H}, \mathrm{d}, \mathrm{J}=6.9 \mathrm{~Hz}, \mathrm{H}_{21}\right), 0.84\left(3 \mathrm{H}, \mathrm{s}, \mathrm{H}_{19}\right), 0.77\left(3 \mathrm{H}, \mathrm{d}, \mathrm{J}=6.1 \mathrm{~Hz}, \mathrm{H}_{27}\right)$; ${ }^{13} \mathrm{C}$ NMR $\left(300 \mathrm{~Hz}, \mathrm{CDCl}_{3}\right)$ : $\square$ 179.4, 170.6, 143.4, 135.0, 106.2, 77.3, 73.1, 66.9, 58.0, 48.6, 46.4, 43.7, 38.9, 37.9, 37.6, 36.8, 36.4, 33.9, 33.3, 31.1, 30.3, 28.7, 27.8, 27.1, 21.4, 17.1, 13.9, 11.9; HRMS (ESI) $m / z$ : calcd 523.2826 $(\mathrm{M}+\mathrm{H})^{+}$, found 523.2820.

Preparation of 8c:

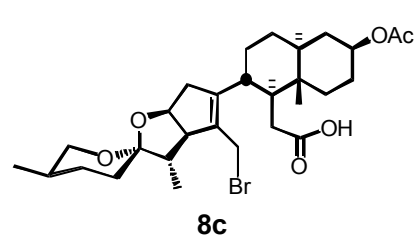

To a solution of compound $3(100 \mathrm{mg}, 0.206 \mathrm{mmol})$ in dichloromethane $(1.0 \mathrm{~mL})$ was added $\mathrm{HBr}(30 \%$ in $\mathrm{AcOH}, 82 \square \mathrm{L}, 0.41$ mmol) at $0{ }^{0} \mathrm{C}$. After stirring at $0-5{ }^{0} \mathrm{C}$ for 2 hours, the solution was quenched with saturated aqueous sodium bicarbonate and diluted with ethyl acetate. The organic layer was washed with brine and dried over anhydrous sodium sulfate. The solvent was removed to give a colorless foam (117 mg, containing $90 \%$ of compound $8 \mathbf{c}$ by ${ }^{1} \mathrm{H}$ NMR).

${ }^{1} \mathrm{H}$ NMR $\left(300 \mathrm{~Hz}, \mathrm{CDCl}_{3}\right): \square 4.65\left(1 \mathrm{H}, \mathrm{m}, \mathrm{H}_{3}\right), 4.49\left(1 \mathrm{H}\right.$, apt, $\left.\mathrm{J}=6.8 \mathrm{~Hz}, \mathrm{H}_{16}\right), 4.05(1 \mathrm{H}$, $\left.\mathrm{d}, \mathrm{J}=10.1 \mathrm{~Hz}, \mathrm{H}_{18 \mathrm{a}}\right), 3.89\left(1 \mathrm{H}, \mathrm{d}, \mathrm{J}=10.1 \mathrm{~Hz}, \mathrm{H}_{18 \mathrm{~b}}\right), 3.46-3.43\left(1 \mathrm{H}, \mathrm{m}, \mathrm{H}_{26}\right), 3.35(1 \mathrm{H}, \mathrm{apt}, \mathrm{J}=$ $\left.10.6 \mathrm{~Hz}, \mathrm{H}_{26 \square}\right), 3.06\left(1 \mathrm{H}\right.$, apt, J = 7.2 Hz, H$\left.{ }_{17}\right), 2.60\left(1 \mathrm{H}, \mathrm{dd}, \mathrm{J}=18.3,7.3 \mathrm{~Hz}, \mathrm{H}_{11}\right), 2.47-2.38$ $(1 \mathrm{H}, \mathrm{m}), 2.34\left(1 \mathrm{H}, \mathrm{d}, \mathrm{J}=18.5 \mathrm{~Hz}, \mathrm{H}_{11}\right), 2.24(1 \mathrm{H}, \mathrm{dd}, \mathrm{J}=17.1,7.0 \mathrm{~Hz}), 1.97\left(3 \mathrm{H}, \mathrm{s}, \mathrm{H}_{3 \mathrm{OAc}}\right)$, $1.09\left(3 \mathrm{H}, \mathrm{d}, \mathrm{J}=6.9 \mathrm{~Hz}, \mathrm{H}_{21}\right), 0.84\left(3 \mathrm{H}, \mathrm{s}, \mathrm{H}_{19}\right), 0.75\left(3 \mathrm{H}, \mathrm{d}, \mathrm{J}=6.1 \mathrm{~Hz}, \mathrm{H}_{27}\right) ;{ }^{13} \mathrm{C} \mathrm{NMR}(300 \mathrm{~Hz}$, $\left.\mathrm{CDCl}_{3}\right): \square 179.8,170.6,143.8,135.1,106.2$, 77.2, 73.0, 66.8, 58.1, 48.5, 46.5, 43.7, 37.9, 37.6, $36.8,36.3,33.8,33.3,31.0,30.8,30.2,28.7,27.8,27.0,26.8,21.3,17.1,13.96,11.9$; HRMS (ESI) $m / z$ : calcd $567.2321(\mathrm{M}+\mathrm{H})^{+}$, found 567.2326 .

\section{Preparation of $\mathbf{8 d :}$}

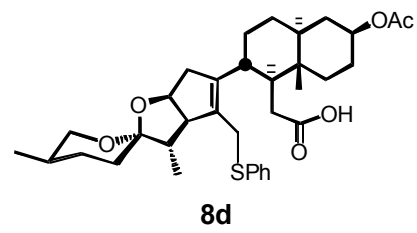

To a solution of compound $\mathbf{3}(60 \mathrm{mg}, 0.12 \mathrm{mmol})$ in dichloromethane $(1.0 \mathrm{~mL})$ was added benzene thiol $(39 \square \mathrm{L}, 0.36 \mathrm{mmol})$ and $p$ toluenesulfonic acid monohydrate $(2.4 \mathrm{mg}, 0.012 \mathrm{mmol})$. After stirring at room temperature for 76 hours, the solution was quenched with saturated aqueous sodium bicarbonate and diluted with ethyl acetate. The organic layer was washed with brine and dried over anhydrous sodium sulfate. The solvent was removed and the residue was chromatographed (hexanes/ethyl acetate $4: 1,2: 1,1: 1)$ to give compound $\mathbf{8 d}(54 \mathrm{mg}, \mathrm{y}=73 \%)$ and starting material 3 (5 mg).

${ }^{1} \mathrm{H}$ NMR $\left(300 \mathrm{~Hz}, \mathrm{CDCl}_{3}\right)$ : $\square 7.41-7.14(5 \mathrm{H}, \mathrm{m}), 4.68-4.67\left(1 \mathrm{H}, \mathrm{m}, \mathrm{H}_{3}\right), 4.55(1 \mathrm{H}, \mathrm{apt}, \mathrm{J}=$ $\left.7.2 \mathrm{~Hz}, \mathrm{H}_{16}\right), 3.71\left(1 \mathrm{H}, \mathrm{d}, \mathrm{J}=12.3 \mathrm{~Hz}, \mathrm{H}_{18 \mathrm{a}}\right), 3.52-3.38\left(3 \mathrm{H}, \mathrm{m}, \mathrm{H}_{18 \mathrm{~b}}, \mathrm{H}_{26 \mathrm{a}}, \mathrm{H}_{26 \mathrm{~b}}\right), 3.11(1 \mathrm{H}$, apt, J 
= 7.2 Hz, $\left.\mathrm{H}_{17}\right), 2.56\left(1 \mathrm{H}, \mathrm{dd}, \mathrm{J}=17.7 \mathrm{~Hz}, 7.3 \mathrm{~Hz}, \mathrm{H}_{11}\right), 2.39-2.24\left(2 \mathrm{H}, \mathrm{m}, \mathrm{H}_{11 \square}\right.$ and $\left.\mathrm{H}_{20}\right), 2.02$ $\left(3 \mathrm{H}, \mathrm{s}, \mathrm{H}_{\mathrm{OAc}}\right), 1.12\left(3 \mathrm{H}, \mathrm{d}, \mathrm{J}=6.9 \mathrm{~Hz}, \mathrm{H}_{21}\right), 0.81\left(3 \mathrm{H}, \mathrm{d}, \mathrm{J}=6.2 \mathrm{~Hz}, \mathrm{H}_{27}\right), 0.78\left(3 \mathrm{H}, \mathrm{s}, \mathrm{H}_{19}\right) ;{ }^{13} \mathrm{C}$ NMR $\left(300 \mathrm{~Hz}, \mathrm{CDCl}_{3}\right)$ : $\square$ 179.6, 170.6, 140.9, 136.7, 134.1, 130.8, 128.98, 126.7, 106.2, 77.5, 73.1, 66.9, 58.9, 48.7, 46.4, 43.7, 37.6, 37.4, 36.7, 36.4, 33.9, 33.0, 32.1, 31.2, 30.3, 28.8, 27.9, 27.1, 21.4, 17.1, 14.1, 11.9; HRMS (ESI) $m / z$ : calcd $597.3250(\mathrm{M}+\mathrm{H})^{+}$, found 597.3245 .

Preparation of $8 \mathrm{e}$ :

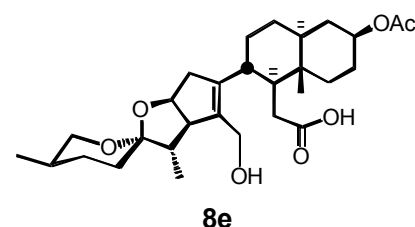

To a solution of 4 (65 mg, $0.12 \mathrm{mmol})$ in methanol $(1 \mathrm{~mL})$ was added $\mathrm{KHCO}_{3}(12 \mathrm{mg}, 0.12 \mathrm{mmol})$. After stirring at room temperature for 12 hours, the reaction was diluted with dichloromethane and washed with brine, and dried over anhydrous sodium sulfate. The solvent was removed and the residue was chromatographed to give $8 \mathbf{e}(55 \mathrm{mg}, \mathrm{y}=$ 91\%) as a white solid. $\mathrm{mp} 108.0 \sim 112.0^{\circ} \mathrm{C}$.

${ }^{1} \mathrm{H}$ NMR $\left(300 \mathrm{~Hz}, \mathrm{CDCl}_{3}\right)$ : $\square$ 4.69-4.63 (1H, m, $\left.\mathrm{H}_{3}\right), 4.46-4.39\left(2 \mathrm{H}, \mathrm{m}, \mathrm{H}_{18 \mathrm{a}}\right.$ and $\left.\mathrm{H}_{16}\right)$, $3.92\left(1 \mathrm{H}, \mathrm{d}, \mathrm{J}=12.0 \mathrm{~Hz}, \mathrm{H}_{18 \mathrm{~b}}\right), 3.55-3.53\left(1 \mathrm{H}, \mathrm{m}, \mathrm{H}_{26 \square}\right), 3.34\left(1 \mathrm{H}\right.$, apt, J = $\left.10.8 \mathrm{~Hz}, \mathrm{H}_{26 \square}\right), 2.97$ $\left(1 \mathrm{H}\right.$, apt, $\left.\mathrm{J}=7.5 \mathrm{~Hz}, \mathrm{H}_{17}\right), 2.62-2.27(4 \mathrm{H}, \mathrm{m}), 1.98\left(3 \mathrm{H}, \mathrm{s}, \mathrm{H}_{3 \mathrm{OAc}}\right), 1.07\left(3 \mathrm{H}, \mathrm{d}, \mathrm{J}=6.9 \mathrm{~Hz}, \mathrm{H}_{21}\right)$, $0.78\left(3 \mathrm{H}, \mathrm{s}, \mathrm{H}_{19}\right), 0.76\left(3 \mathrm{H}, \mathrm{d}, \mathrm{J}=6.5 \mathrm{~Hz}, \mathrm{H}_{27}\right) ;{ }^{13} \mathrm{C} \mathrm{NMR}\left(300 \mathrm{~Hz}, \mathrm{CDCl}_{3}\right): \square$ 176.4, 170.7, $140.2,137.1,106.7,77.5,73.1,66.6,57.1,56.7,48.2,46.9,43.6,38.3,38.2,36.7,36.3,35.1$, 33.7, 31.04, 31.0, 30.1, 28.6, 27.9, 27.2, 21.3, 17.0, 13.8, 11.9; HRMS (ESI) $\mathrm{m} / \mathrm{z}$ : calcd 527.2985 $(\mathrm{M}+\mathrm{Na})^{+}$, found 527.2981 .

Standard procedure for PPSE promoted Friedel-Crafts reaction:

A solution of $\mathrm{P}_{2} \mathrm{O}_{5}$ (25 equivalent) and hexamethyldisiloxane (37.5 equivalent) in dichloroethane (1.8 x volume of hexamethyldisiloxane, dried over $\left.\mathrm{K}_{2} \mathrm{CO}_{3}\right)$ was heated at reflux for 1.5 hours. Acid ( 1 equivalent) in dichloroethane $(0.2 \mathrm{M})$ was added in a small stream to the above clear, colorless solution while keeping the solution at reflux. The reaction mixture was heated at reflux for another 1 to 2 hours. The solution was cooled to room temperature using an ice bath and was then diluted with dichloromethane, washed with cold $10 \% \mathrm{NaOH}$, brine, and dried over anhydrous sodium sulfate. The solvent was removed and the residue was chromatographed to give the desired cyclized product.

\section{Preparation of 9a:}

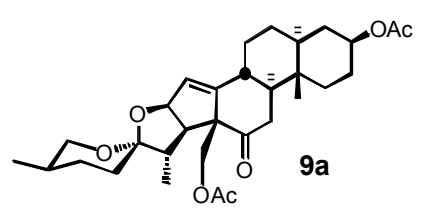

Following the above procedure, $66 \mathrm{mg}$ of $9 \mathrm{a}(\mathrm{y}=70 \%), 6.5 \mathrm{mg}$ of $7(\mathrm{y}$ $=8 \%$ ), and $5 \mathrm{mg}$ of $\mathbf{3}(\mathrm{y}=5 \%)$ were obtained starting from $100 \mathrm{mg}$ of acid $\mathbf{8 a}$ (containing $3 \%$ of compound 3 ) after silica gel chromatography (toluene/ethyl acetate 10:1 to $2: 1$ ).

Compound 9a: mp 192.0 193.8 ${ }^{0} \mathrm{C}$. ${ }^{1} \mathrm{H}$ NMR $(300 \mathrm{~Hz}$, $\left.\mathrm{CDCl}_{3}\right)$ : $\square 5.52\left(1 \mathrm{H}, \mathrm{s}, \mathrm{H}_{15}\right), 4.75-4.71\left(1 \mathrm{H}, \mathrm{m}, \mathrm{H}_{16}\right), 4.72\left(1 \mathrm{H}, \mathrm{d}, \mathrm{J}=12.0 \mathrm{~Hz}, \mathrm{H}_{18 \mathrm{a}}\right), 4.68-4.57$ $\left(1 \mathrm{H}, \mathrm{m}, \mathrm{H}_{3}\right), 4.03\left(1 \mathrm{H}, \mathrm{d}, \mathrm{J}=12.0 \mathrm{~Hz}, \mathrm{H}_{18 \mathrm{~b}}\right), 3.50-3.46\left(1 \mathrm{H}, \mathrm{m}, \mathrm{H}_{26}\right), 3.40-3.32\left(2 \mathrm{H}, \mathrm{m}, \mathrm{H}_{26}\right.$ and $\left.\mathrm{H}_{17}\right), 2.53\left(1 \mathrm{H}\right.$, apt, J = 14.2 Hz), 2.41-2.34 (2H, m), $1.983\left(3 \mathrm{H}, \mathrm{s}, \mathrm{H}_{3 \mathrm{Ac}}\right), 1.977$ (3H, s, $\mathrm{H}_{18 \mathrm{OAc}}$ ), $1.03\left(3 \mathrm{H}, \mathrm{d}, \mathrm{J}=6.7 \mathrm{~Hz}, \mathrm{H}_{21}\right), 0.91\left(3 \mathrm{H}, \mathrm{s}, \mathrm{H}_{19}\right), 0.76\left(3 \mathrm{H}, \mathrm{d}, \mathrm{J}=6.3 \mathrm{~Hz}, \mathrm{H}_{27}\right) ;{ }^{13} \mathrm{C} \mathrm{NMR}(300 \mathrm{~Hz}$, $\left.\mathrm{CDCl}_{3}\right): \square 207.6,170.53,170.51,150.9,123.8,107.4,83.7,72.9,67.0,66.8,65.1,53.7,49.1$, 44.4, 43.9, 38.0, 36.3, 36.1, 35.4, 33.6, 31.3, 30.2, 29.5, 28.7, 27.7, 27.1, 21.3, 20.7, 17.1, 13.5, 11.7; HRMS (ESI) $m / z$ : calcd $529.3165(\mathrm{M}+\mathrm{H})^{+}$, found 529.3156 . 


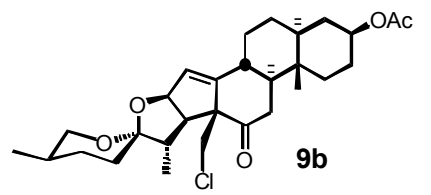

Starting from $100 \mathrm{mg}$ of acid $\mathbf{8 b}$, following the same procedure, $81 \mathrm{mg}$ of 9b $(y=84 \%)$ was obtained as a white solid after silica gel chromatography (hexanes/ethyl acetate 5:1 to $3: 1$ ). $\operatorname{mp} 240.0 \sim 242.6{ }^{0} \mathrm{C}$.

${ }^{1} \mathrm{H}$ NMR $\left(300 \mathrm{~Hz}, \mathrm{CDCl}_{3}\right)$ : $\square 5.54\left(1 \mathrm{H}, \mathrm{s}, \mathrm{H}_{15}\right), 4.70(1 \mathrm{H}, \mathrm{dd}, \mathrm{J}$ $\left.=8.1 \mathrm{~Hz}, 2.1 \mathrm{~Hz}, \mathrm{H}_{16}\right), 4.67-4.58\left(1 \mathrm{H}, \mathrm{m}, \mathrm{H}_{3}\right), 3.96\left(1 \mathrm{H}, \mathrm{d}, \mathrm{J}=12.2 \mathrm{~Hz}, \mathrm{H}_{18 \mathrm{a}}\right), 3.62(1 \mathrm{H}, \mathrm{d}, \mathrm{J}=$ $\left.12.2 \mathrm{~Hz}, \mathrm{H}_{18 \mathrm{~b}}\right), 3.48\left(1 \mathrm{H}, \mathrm{dd}, \mathrm{J}=10.7 \mathrm{~Hz}, 2.3 \mathrm{~Hz}, \mathrm{H}_{26 \square}\right), 3.41-3.32\left(2 \mathrm{H}, \mathrm{m}, \mathrm{H}_{26 \square}\right.$ and $\left.\mathrm{H}_{17}\right), 2.46-$ $2.33(3 \mathrm{H}, \mathrm{m}), 1.98\left(3 \mathrm{H}, \mathrm{s}, \mathrm{H}_{3 \mathrm{OAc}}\right), 1.13\left(3 \mathrm{H}, \mathrm{d}, \mathrm{J}=6.7 \mathrm{~Hz}, \mathrm{H}_{21}\right), 0.91\left(3 \mathrm{H}, \mathrm{s}, \mathrm{H}_{19}\right), 0.76(3 \mathrm{H}, \mathrm{d}, \mathrm{J}$ $\left.=6.3 \mathrm{~Hz}, \mathrm{H}_{27}\right) ;{ }^{13} \mathrm{C} \mathrm{NMR}\left(300 \mathrm{~Hz}, \mathrm{CDCl}_{3}\right): \square 206.3,170.5,151.7,124.0,107.6,83.3,72.8,68.4$, 66.96, 53.6, 49.2, 45.4, 44.2, 43.8, 37.97, 36.3, 36.1, 35.3, 33.6, 31.3, 30.1, 29.4, 28.7, 27.7, 27.0, 21.3, 17.0, 14.0, 11.7; HRMS (ESI) $m / z$ : calcd 505.2721 $(\mathrm{M}+\mathrm{H})^{+}$, found 505.2721.

Preparation of 9c:

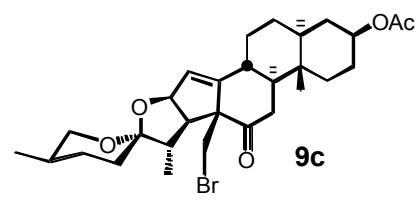

Starting from $117 \mathrm{mg}$ of acid $\mathbf{8 c}$ (90\% purity based on ${ }^{1} \mathrm{H}$ NMR), following the same procedure, $79 \mathrm{mg}$ of $9 \mathrm{c}(\mathrm{y}=78 \%)$ was obtained as a white solid after silica gel chromatography (hexanes/ethyl acetate 5:1 to $3: 1)$. mp $180.0 \sim 181.6^{\circ} \mathrm{C}$.

${ }^{1} \mathrm{H}$ NMR $\left(300 \mathrm{~Hz}, \mathrm{CDCl}_{3}\right): \square 5.55\left(1 \mathrm{H}, \mathrm{s}, \mathrm{H}_{15}\right), 4.69(1 \mathrm{H}, \mathrm{dd}, \mathrm{J}$ $\left.=8.1 \mathrm{~Hz}, 2.2 \mathrm{~Hz}, \mathrm{H}_{16}\right), 4.67-4.57\left(1 \mathrm{H}, \mathrm{m}, \mathrm{H}_{3}\right), 3.77\left(1 \mathrm{H}, \mathrm{d}, \mathrm{J}=11.4 \mathrm{~Hz}, \mathrm{H}_{18 \mathrm{a}}\right), 3.50-3.31(4 \mathrm{H}, \mathrm{m}$, $\mathrm{H}_{26 \square}, \mathrm{H}_{26 \square}, \mathrm{H}_{18 \mathrm{~b}}$ and $\left.\mathrm{H}_{17}\right), 2.45-2.34(3 \mathrm{H}, \mathrm{m}), 1.98\left(3 \mathrm{H}, \mathrm{s}, \mathrm{H}_{3 \mathrm{Ac}}\right), 1.15\left(3 \mathrm{H}, \mathrm{d}, \mathrm{J}=6.9 \mathrm{~Hz}, \mathrm{H}_{21}\right)$, $0.91\left(3 \mathrm{H}, \mathrm{s}, \mathrm{H}_{19}\right), 0.75\left(3 \mathrm{H}, \mathrm{d}, \mathrm{J}=6.3 \mathrm{~Hz}, \mathrm{H}_{27}\right) ;{ }^{13} \mathrm{C} \mathrm{NMR}\left(300 \mathrm{~Hz}, \mathrm{CDCl}_{3}\right): \square$ 206.5, 170.5, $152.5,123.9,107.7,83.2,72.8,67.6,66.98,53.5,49.5,44.0,43.8,37.9,36.3,36.1,35.3,33.63$, 33.60, 31.4, 30.1, 29.4, 28.7, 27.7, 27.1, 21.3, 17.1, 14.4, 11.7; HRMS (ESI) m/z: calcd 549.2216 $(\mathrm{M}+\mathrm{H})^{+}$, found 549.2213 .

Preparation of 9d:

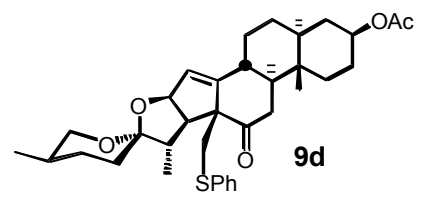

Starting from $90 \mathrm{mg}$ of acid $\mathbf{8 d}$, following the same procedure, $65 \mathrm{mg}$ of 9d $(y=74 \%)$ was obtained as a white fluffy solid after silica gel chromatography (hexanes/ethyl acetate 6:1, 5:1).

${ }^{1} \mathrm{H}$ NMR $\left.\left(300 \mathrm{~Hz}, \mathrm{CDCl}_{3}\right): \square 7.35-7.1995 \mathrm{H}, \mathrm{m}\right), 5.58(1 \mathrm{H}, \mathrm{s}$, $\left.\mathrm{H}_{15}\right), 4.76\left(1 \mathrm{H}, \mathrm{dd}, \mathrm{J}=7.9 \mathrm{~Hz}, 2.1 \mathrm{~Hz}, \mathrm{H}_{16}\right), 4.71-4.60\left(1 \mathrm{H}, \mathrm{m}, \mathrm{H}_{3}\right)$, 3.55-3.51 (1H, m, $\left.\mathrm{H}_{26}\right), 3.48-3.33\left(3 \mathrm{H}, \mathrm{m}, \mathrm{H}_{26}, \mathrm{H}_{18 \mathrm{a}}\right.$ and $\left.\mathrm{H}_{17}\right), 3.21\left(1 \mathrm{H}, \mathrm{d}, \mathrm{J}=12.6 \mathrm{~Hz}, \mathrm{H}_{18 \mathrm{~b}}\right)$, 2.43-2.28 (3H, m), $2.02\left(3 \mathrm{H}, \mathrm{s}, \mathrm{H}_{\mathrm{OAc}}, 1.19\left(3 \mathrm{H}, \mathrm{d}, \mathrm{J}=6.9 \mathrm{~Hz}, \mathrm{H}_{21}\right), 0.81\left(3 \mathrm{H}, \mathrm{d}, \mathrm{J}=6.3 \mathrm{~Hz}, \mathrm{H}_{27}\right)\right.$, $0.78\left(3 \mathrm{H}, \mathrm{s}, \mathrm{H}_{19}\right) ;{ }^{13} \mathrm{C} \mathrm{NMR}\left(300 \mathrm{~Hz}, \mathrm{CDCl}_{3}\right)$ : $\square 208.3,170.5,153.4,136.8,129.7,129.1,126.5$, $122.9,107.7,83.6,72.9,66.98,66.9,53.2,50.1,44.3,43.8,39.1,38.1,36.2,36.1,35.2,33.6$, 31.4, 30.2, 29.3, 28.7, 27.8, 27.1, 21.3, 17.1, 14.3, 11.5; HRMS (ESI) $\mathrm{m} / z$ : calcd 579.3144 $(\mathrm{M}+\mathrm{H})^{+}$, found 579.3146 .

Weak acid catalyzed equilibrium, preparation of $\mathbf{1 3}$ :

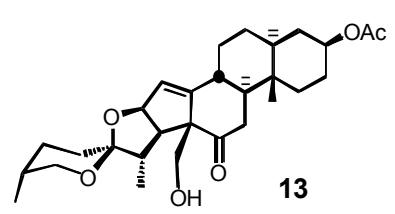

To a solution of $9 \mathrm{e}(100 \mathrm{mg}, 0.21 \mathrm{mmol})$ in dichloromethane was added PPTS $(3 \mathrm{mg}, 0.01 \mathrm{mmol})$. After stirring at room temperature for 5 hours, the reaction was quenched with saturated sodium bicarbonate and washed with brine once. The organic layers were dried over anhydrous sodium sulfate. The solvent was removed to give a white solid. ${ }^{1} \mathrm{H}$

NMR showed that the ratio of $\mathbf{9 e : 1 2 : 1 3}$ was 67:28:5. Several reactions were performed following the same procedure but used different solvents $\left(\mathrm{CH}_{3} \mathrm{OH}\right.$, or $\mathrm{CH}_{3} \mathrm{OH} / \mathrm{CH}_{2} \mathrm{Cl}_{2}$, or $75 \%$ aqueous $\mathrm{AcOH} / \mathrm{CH}_{2} \mathrm{Cl}_{2}$ ). More experiments were performed using 12 as starting material to get the ${ }^{1} \mathrm{H}$ NMR ratios. Some of the recovered products were combined and chromatographed (hexanes/ethyl acetate, $3: 1,1.5: 1,100 \%$ ethyl acetate) to give $20 \mathrm{mg}$ of $13 . \mathrm{mp} 190.4 \sim 194.0{ }^{0} \mathrm{C}$. 
${ }^{1} \mathrm{H}$ NMR $\left(300 \mathrm{~Hz}, \mathrm{CDCl}_{3}\right): \square 5.53\left(1 \mathrm{H}, \mathrm{s}, \mathrm{H}_{15}\right), 5.14\left(1 \mathrm{H}, \mathrm{d}, \mathrm{J}=10.7 \mathrm{~Hz}, \mathrm{H}_{18-\mathrm{OH}}\right), 5.03$ $\left(1 \mathrm{H}, \mathrm{dd}, \mathrm{J}=8.4 \mathrm{~Hz}, 1.9 \mathrm{~Hz}, \mathrm{H}_{16}\right)$, 4.69-4.59 $\left(1 \mathrm{H}, \mathrm{m}, \mathrm{H}_{3}\right), 3.97-3.90\left(2 \mathrm{H}, \mathrm{m}, \mathrm{H}_{18 \mathrm{a}}\right.$ and $\left.\mathrm{H}_{26 \square}\right), 3.68$ $\left(1 \mathrm{H}, \mathrm{d}, \mathrm{J}=11.2 \mathrm{~Hz}, \mathrm{H}_{18 \mathrm{~b}}\right), 3.35\left(1 \mathrm{H}, \mathrm{d}, \mathrm{J}=8.4 \mathrm{~Hz}, \mathrm{H}_{17}\right), 3.13\left(1 \mathrm{H}, \mathrm{bd}, \mathrm{J}=11.1 \mathrm{~Hz}, \mathrm{H}_{26}\right), 2.60-$ $2.51(2 \mathrm{H}, \mathrm{m}), 2.36\left(1 \mathrm{H}, \mathrm{dd}, \mathrm{J}=15.4 \mathrm{~Hz}, 5.0 \mathrm{~Hz}, \mathrm{H}_{11 \square}\right), 2.27\left(1 \mathrm{H}\right.$, apq, J $\left.=7.3 \mathrm{~Hz}, \mathrm{H}_{20}\right), 1.99(3 \mathrm{H}$, $\left.\mathrm{s}, \mathrm{H}_{3-\mathrm{OAc}}\right), 1.06\left(3 \mathrm{H}, \mathrm{d}, \mathrm{J}=7.3 \mathrm{~Hz}, \mathrm{H}_{21}\right), 1.01\left(3 \mathrm{H}, \mathrm{d}, \mathrm{J}=7.0 \mathrm{~Hz}, \mathrm{H}_{27}\right), 0.91\left(3 \mathrm{H}, \mathrm{s}, \mathrm{H}_{19}\right) ;{ }^{13} \mathrm{C}$ NMR (300 Hz, $\left.\mathrm{CDCl}_{3}\right): \square 208.8,170.6,150.9,124.5,108.8,86.7,73.1,71.7,65.4,62.9,53.4$, 49.8, 44.4, 44.1, 37.7, 36.3, 36.2, 34.97, 33.7, 29.6, 27.7, 27.2, 27.0, 25.7, 25.6, 21.4, 18.4, 16.3, 11.7; HRMS (ESI) $m / z$ : calcd 509.2879 (M+Na) ${ }^{+}$, found 509.2883. 